Supporting Information:

\title{
New Surfactants from Biobased Materials
}

Michael A. Jackson, ${ }^{* a}$ Kervin O. Evans, ${ }^{a}$ Neil P.J. Price, ${ }^{a}$ Judith A. Blackburn, ${ }^{a}$ Karen J. Ray ${ }^{a}$, Charles J.

Ward $^{\mathrm{a}}$, and Karl E. Vermillion ${ }^{\mathrm{b}}$

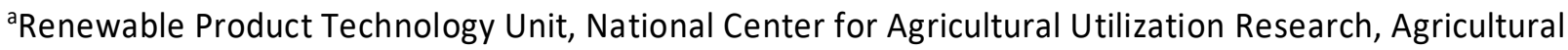

Research Service, United States Department of Agriculture. 1815 N. University St. Peoria, IL 61604 USA

${ }^{\mathrm{b}}$ Functional Foods Research Unit, National Center for Agricultural Utilization Research, Agricultural

Research Service, United States Department of Agriculture. 1815 N. University St. Peoria, IL 61604 USA

Number of pages 7

Number of figures 4

Number of tables 1 


\section{Materials and Methods}

The 2-undecanone and the sugars used in this work were obtained from Millipore-Sigma.

Anhydrous ammonia was from Airgas. $5 \mathrm{wt} \% \mathrm{Rh} / \mathrm{Al}_{2} \mathrm{O}_{3}$ and $5 \mathrm{wt} \% \mathrm{Rh} / \mathrm{C}$ were from Engelhard and were used without a prereduction step. Methanol was from Fisher Scientific.

General methods for the preparation of $C$-glycoside ketones. The conversion of sugars to the $C$ glycoside ketones was performed as described in the literature. ${ }^{1-2}$ Briefly, the sugars were treated at 85 ${ }^{\circ} \mathrm{C}$ with a $20 \%$ molar excess of acetyl acetone and $\mathrm{NaHCO}_{3}$. After about $4 \mathrm{~h}$ the solution was treated with Dowex cation exchange resin to remove $\mathrm{Na}^{+}$. The resin was removed by filtration and the filtrate was taken to dryness using a rotary evaporator operating at $65^{\circ} \mathrm{C}$. Finally, the last of the water was removed under vacuum at $50{ }^{\circ} \mathrm{C}$ to a residual pressure under $0.1 \mathrm{mbar}$.

\section{Synthesis of the side product bis(maltose $C$-glycoside)amine}

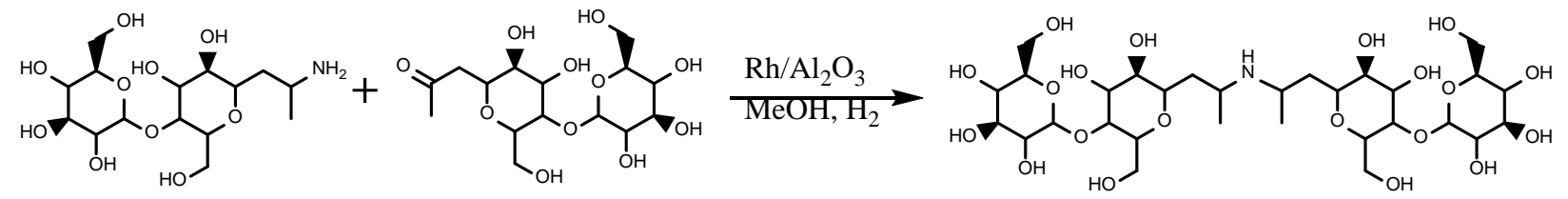

Figure S1. Scheme showing the formation of the maltosyl diamine.

A $30 \mathrm{ml} \mathrm{HEL} \mathrm{stirred} \mathrm{reactor} \mathrm{was} \mathrm{charged} \mathrm{with} 500 \mathrm{mg}$ maltose $C$-glycoside amine and $500 \mathrm{mg}$ maltose $C$ glycoside ketone (1.3 mmol each), $250 \mathrm{mg} 5 \mathrm{wt} \% \mathrm{Rh} / \mathrm{Al}_{2} \mathrm{O}_{3}$, and $15 \mathrm{ml}$ methanol. The reactor was heated to $65^{\circ} \mathrm{C}$ and charged with 1.3 bar hydrogen pressure and stirred at $500 \mathrm{rpm}$ for $3 \mathrm{~h}$. The product was isolated by removal of the catalyst by filtration and removal of the solvent under reduced pressure. MALDI-TOF MS: $m / z 750,[\mathrm{M}+\mathrm{H}]^{+}$. 


\section{Detailed NMR data}

Glucosyl C-glycoside ketone (1a). ${ }^{1} \mathrm{H}$ NMR $\left(500 \mathrm{MHz}, \mathrm{D}_{2} \mathrm{O}\right) \delta 3.80(\mathrm{~m}, 1 \mathrm{H}), 3.60(\mathrm{~m}, 1 \mathrm{H}), 3.70(\mathrm{~m}, 1 \mathrm{H})$, $3.40(\mathrm{t}, J=8.9 \mathrm{~Hz}, 1 \mathrm{H}), 3.30(\mathrm{~m}, 2 \mathrm{H}), 3.1(\mathrm{t}, 1 \mathrm{H}), 2.95(\mathrm{dd}, J=3.2, J=16 \mathrm{~Hz}, 1 \mathrm{H}), 2.66(\mathrm{dd}, J=9.0, J=16 \mathrm{~Hz}, 1 \mathrm{H})$ $2.20(\mathrm{~s}, 3 \mathrm{H}) .{ }^{13} \mathrm{C}$ NMR $\left(125 \mathrm{MHz}, \mathrm{D}_{2} \mathrm{O}\right) \delta 213,79.5,77.3,75.3,73.2,69.9,60.7,45.7$, 30.0. MALDI-TOF MS: $m / z 243,[\mathrm{M}+\mathrm{Na}]^{+}$.

Glucosyl C-glycoside amine (1b). $72 \%$ Yield. ${ }^{1} \mathrm{H} N M R\left(500 \mathrm{MHz}, \mathrm{D}_{2} \mathrm{O}\right.$ acidified with $\left.\mathrm{D}_{2} \mathrm{SO}_{4}\right) . \delta 3.80(\mathrm{~m}$, 1H), $3.60(\mathrm{~m}, 1 \mathrm{H}), 3.70(\mathrm{~m}, 1 \mathrm{H}), 3.32(\mathrm{~m}, 1 \mathrm{H}), 3.30(\mathrm{~m}, 1 \mathrm{H}), 3.18$ and $3.15(\mathrm{t}, J=6.8 \mathrm{~Hz}, 2 \mathrm{H}), 1.81$ and 1.75 (m, J=2.2 Hz, 1H), $1.52(\mathrm{~m}, J=2.2 \mathrm{~Hz}, 1 \mathrm{H}) 1.20(\mathrm{dd}, J=6 \mathrm{~Hz}, 0.6 \mathrm{H}), 1.1$ (dd, J=6 Hz, 2.4H). ${ }^{13} \mathrm{C}$ NMR $(125$ $\mathrm{MHz}, \mathrm{D}_{2} \mathrm{O}$ acidified with $\left.\mathrm{D}_{2} \mathrm{SO}_{4}\right) \delta 79.5,77.8,77.1,73.7,70.0,61.12 \mathrm{x}, 44.5,42.8,39.7,39.3,22.3,20.9$, 20.1. MALDI-TOF MS: $m / z 222,[\mathrm{M}+\mathrm{H}]^{+}, 244,[\mathrm{M}+\mathrm{Na}]^{+}$.

Glucosyl C-glycoside 2-aminoundecane (1c). 90\% yield. ${ }^{1} \mathrm{H}$ NMR $\left(500 \mathrm{MHz}, \mathrm{DMSO}-\mathrm{d}_{6}\right) \delta 3.63(\mathrm{~m}, 1 \mathrm{H})$, $3.39(\mathrm{~m}, 1 \mathrm{H}), 3.09(\mathrm{~m}, 1 \mathrm{H}), 3.02(\mathrm{~m}, 1 \mathrm{H}), 2.84(\mathrm{~m}, 1 \mathrm{H}), 1.43(\mathrm{~m}, 2 \mathrm{H}), 1.24(\mathrm{bs}, 16 \mathrm{H}) 0.93(\mathrm{~m}, 6 \mathrm{H}), 0.85(\mathrm{t}$, $J=7.3 \mathrm{~Hz}, 3 \mathrm{H}) .{ }^{13} \mathrm{C}$ NMR $\left(125 \mathrm{MHz}\right.$, DMSO- $\left.d_{6}\right) \delta 80.8,79.0,75.0,71.0,62.02 x, 32.0,29.6,25.7,24.0,22.8$, 21.9, 21.4, 14.5. MALDI-TOF MS: $m / z 376,[\mathrm{M}+\mathrm{H}]^{+}$.

Maltosyl C-glycoside ketone (2a). ${ }^{1} \mathrm{H} N M R\left(500 \mathrm{MHz}, \mathrm{D}_{2} \mathrm{O}\right) . \quad \delta 5.33,5.28(\mathrm{~d}, \mathrm{~J}=3.9 \mathrm{~Hz}, 1 \mathrm{H}), 3.83,3.79$, 3.73, 3.72, 3.71, 3.70, 3.65, 3.55, 3.52, 3.48, 3.36 (overlapping $m, 14 \mathrm{H}), 3.36(\mathrm{t}, \mathrm{J}=9.5 \mathrm{~Hz}, 1.4 \mathrm{H}), 3.20(\mathrm{t}$, $J=9.5 \mathrm{~Hz}, 0.6 \mathrm{H}$ ), 2.96, 2.94, 2.92, 2.87 (overlapping $m, 1.4 \mathrm{H}$ ), 2.66 (dd, J=9.3 Hz, 0.6H), $2.22(\mathrm{~s}, 1.4 \mathrm{H}), 2.21$ (s, 1.8H). ${ }^{13} \mathrm{C}$ NMR $\left(125 \mathrm{MHz} \mathrm{D}_{2} \mathrm{O}\right) \delta 213.2,212.9,99.6,99.5,78.1,77.7,77.0,76.8,75.1,73.0,72.9,72.9$, 72.7, 72.6, 71.7, 71.6, 71.1, 70.2, 69.3, 60.8, 60.5, 45.6, 40.1, 29.9, 29.8. MALDI-TOF MS: $\mathrm{m} / \mathrm{z}$ 405, $[\mathrm{M}+\mathrm{Na}]^{+}$.

Maltosyl C-glycoside amine (2b). $92 \%$ yield. ${ }^{1} \mathrm{H}$ NMR (500 MHz, $\left.\mathrm{D}_{2} \mathrm{O}\right) . \delta 5.33$ (d, J=3.9Hz, $\left.1 \mathrm{H}\right), 3.78,3.69$, 3.67, 3.64, 3.54, 3.52, 3.46, 3.40, 3.39, 3.35 (overlapping $m, 22 H), 1.95(m, 1 H), 1.60(m, 1 H), 1.18(2 d$, $J=6.5 \mathrm{~Hz}, 2 \mathrm{H}) .{ }^{13} \mathrm{C} N M R\left(125 \mathrm{MHz} \mathrm{D}_{2} \mathrm{O}\right) \delta 99.6,77.9,77.7,77.6,77.1,77.0,73.5,72.9,72.7,72.7,71.7$, 
71.7, 69.3, 60.9, 60.9, 60.7, 60.5, 60.5, 48.9, 45.8, 37.3, 19.4. MALDI-TOF MS: $m / z$ 384, $[\mathrm{M}+\mathrm{H}]^{+}, 406$, $[\mathrm{M}+\mathrm{Na}]^{+}$.

Maltosyl C-glycoside 2-aminoundecane (2c). 68\% yield. ${ }^{1} \mathrm{H}$ NMR (500 MHz, D $2 \mathrm{O}$ acidified). 5.34, 5.29 (2 unresolved doublets, $1 \mathrm{H}), 3.85,3.76,3.66,3.65,3.64,3.53,3.52(\mathrm{~m}, 14 \mathrm{H}), 3.36,3.34(\mathrm{~m}, 2.1 \mathrm{H}), 3.17(\mathrm{~m}$, $0.6 \mathrm{H}), 1.60$ (bs, $1 \mathrm{H}), 1.42$ (bs, $1 \mathrm{H}), 1.27,1.23,1.18,1.15$ (bm, 19H), 0.74 (t, J=6.7 Hz, 3H). ${ }^{13} \mathrm{C}$ NMR $(125$ $\left.\mathrm{MHz} \mathrm{D}_{2} \mathrm{O}\right) \delta 99.6,99.1,77.9,77.8,77.5,73.0,72.8,72.7,71.6,71.5,69.2,60.9,60.5,51.9,49.6,32.5$, 31.2, 22.1, 16.3, 15.8, 15.3, 13.4. MALDI-TOF MS: $m / z$ 538, $[\mathrm{M}+\mathrm{H}]^{+}, 560,[\mathrm{M}+\mathrm{Na}]^{+}$. Analyses calculated for $\mathrm{C}_{26} \mathrm{H}_{51} \mathrm{NO}_{10}: \mathrm{C}, 58.08 ; \mathrm{H}, 9.56 ; \mathrm{N}, 2.61$. Found: $\mathrm{C}, 53.95 ; \mathrm{H}, 8.95 ; \mathrm{N}, 2.25$.

Lactosyl C-glycoside ketone (3a). 90\% yield. ${ }^{1} \mathrm{H}$ NMR (500 MHz, $\left.\mathrm{D}_{2} \mathrm{O}\right) . \delta 4.50(\mathrm{~m}, 0.4 \mathrm{H}), 4.38,4.37$ (d, $\mathrm{J}=7.8 \mathrm{~Hz}, 1 \mathrm{H}$ together. These integrate $2: 1), 3.87(\mathrm{dd}, \mathrm{J}=3.3), 3.83,3.74,3.73,3.71,3.70,3.66,3.60,3.57$, 3.49, 3.48 (unresolved group, 13H), $3.22(\mathrm{~m}, 0.6 \mathrm{H}), 2.96$ (dd, J=2.8 Hz, J=16.8 Hz, 0.6H), $2.90(\mathrm{~m}, 0.4 \mathrm{H})$, 2.22, 2.21 (2 singlets, $3 \mathrm{H}) .{ }^{13} \mathrm{C}$ NMR $\left(125 \mathrm{MHz}_{2} \mathrm{O}\right) \delta$ 102.9, 78.7, 78.5, 78.3, 75.8, 75.4, 75.1, 72.8, 72.5, 72.3, 71.7, 71.6, 71.0, 70.1, 68.6, 61.0, 60.1, 45.6, 39.7, 29.8. MALDI-TOF MS: $m / z$ 405, $[\mathrm{M}+\mathrm{Na}]^{+}$. Lactosyl C-glycoside amine (3b). $82 \%$ yield. ${ }^{1} \mathrm{H} N M R\left(500 \mathrm{MHz}, \mathrm{D}_{2} \mathrm{O}\right) . \delta 4.36$, (d J=7.8 Hz, $\left.1 \mathrm{H}\right), 3.87,3.85$, 3.70, 3.64, 3.58, 3.55, 3.53, 3.47, 3.45, 3.44 (overlapping $m, 15 H$ ), 3.19 (ddd, J=3.1, J=9, J=20 Hz, $1 \mathrm{H}$ ), $1.97(m, 1 H), 1.66(m, 1 H), 1.21(d, J=6.7 \mathrm{~Hz}, 3 \mathrm{H}) .{ }^{13} \mathrm{C} N M R\left(125 \mathrm{MHz} \mathrm{D}_{2} \mathrm{O}\right) \delta$ 102.9, 78.6, 78.5, 78.3, 78.1, $77.4,75.8,75.7,73.2,72.9,72.5,71.0,68.6,61.0,60.3,60.2,48.9,46.2,44.4,36.6,36.5,19.4,18.9$. MALDI-TOF MS: $m / z$ 384, $[\mathrm{M}+\mathrm{H}]^{+}, 406,[\mathrm{M}+\mathrm{Na}]^{+}$. Lactosyl C-glycoside 2-aminoundecane (3c). $72 \%$ yield. ${ }^{1} \mathrm{H} N M R\left(500 \mathrm{MHz}, \mathrm{D}_{2} \mathrm{O}\right.$, acidified). $\delta 4.31$ (dt, $J=7.8 \mathrm{~Hz}, 1 \mathrm{H}), 3.84,3.81,3.65,3.61,3.60,3.54,3.50,3.46,3.42,3.29,3.17(\mathrm{~m}, 17 \mathrm{H}), 2.1-1.7(\mathrm{~m}, 2 \mathrm{H}), 1.59$ $(m, 1 H), 1.42,1.27(t, J=5.7 \mathrm{~Hz}, 3 \mathrm{H}), 1.16(\mathrm{~m}, 15 \mathrm{H}), 0.74(\mathrm{t}, J=7.1 \mathrm{~Hz}, 3 \mathrm{H}) .{ }^{13} \mathrm{C} N M R\left(125 \mathrm{MHz} \mathrm{D}_{2} \mathrm{O}\right) \delta$ $102.9,102.8,78.6,78.5,78.4,78.2,78.0,75.6,75.3,73.1,72.9,72.5,70.9,68.5,61.0,60.3,51.9,49.5$, 32.6, 31.2, 28.6, 28.5, 28.4, 28.3, 22.0, 16.2, 15.8, 15.6, 15.3, 13.4. MALDI-TOF MS: $m / z 538,[M+H]^{+}$, 
$560,[\mathrm{M}+\mathrm{Na}]^{+}$. Analysis calculated for $\mathrm{C}_{26} \mathrm{H}_{51} \mathrm{NO}_{10}: \mathrm{C}, 58.08 ; \mathrm{H}, 9.56 ; \mathrm{N}, 2.61$. Found: $\mathrm{C}, 52.69 ; \mathrm{H}, 9.05 ; \mathrm{N}$, 2.18 .

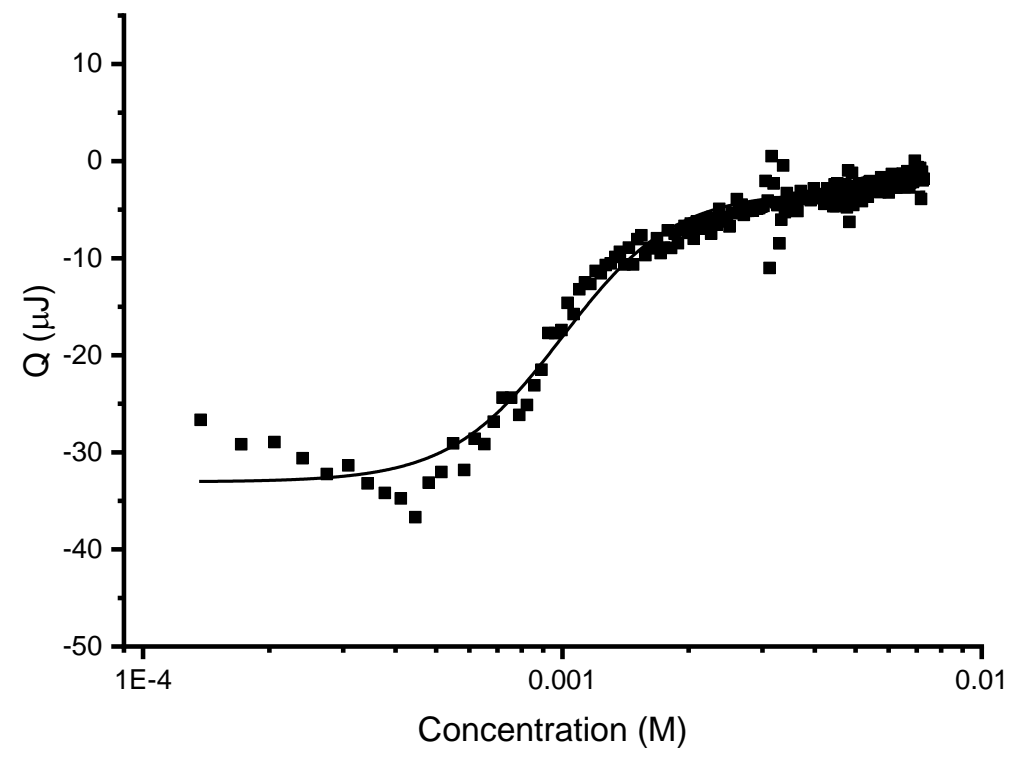

Figure S2. ITC plot used to determine the CMC of glucosyl-C-2-aminoundecane, 1c.

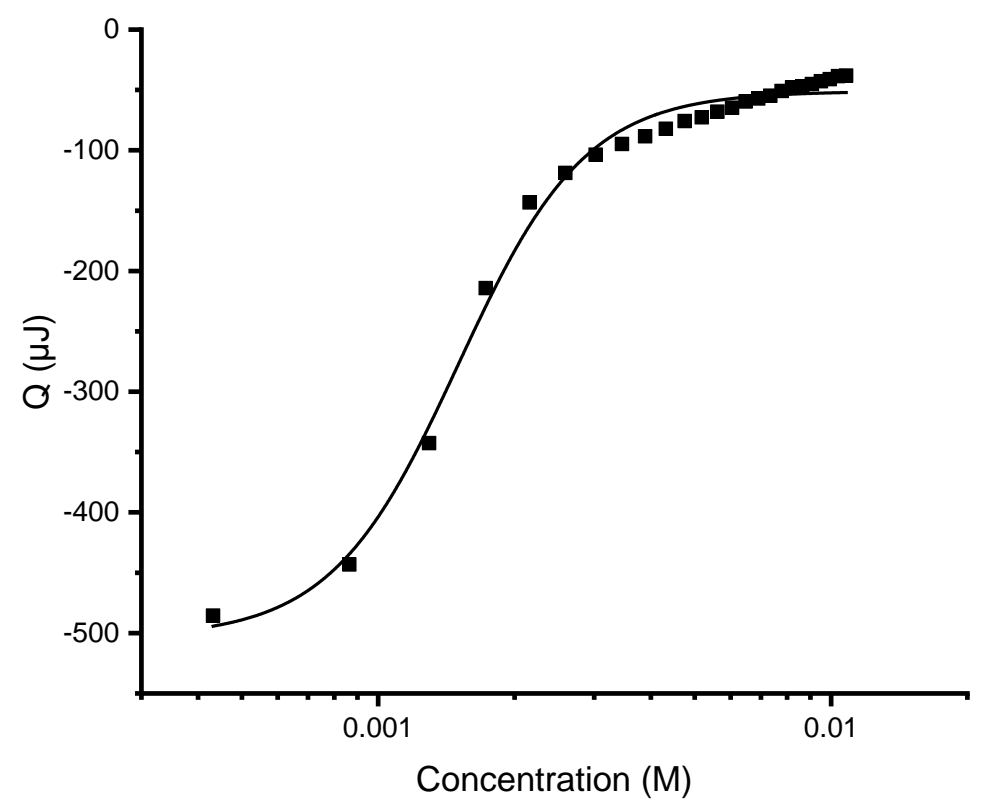

Figure S3. ITC plot used to determine the CMC of maltosyl-C-2-aminoundecane, $\mathbf{2 c}$. 


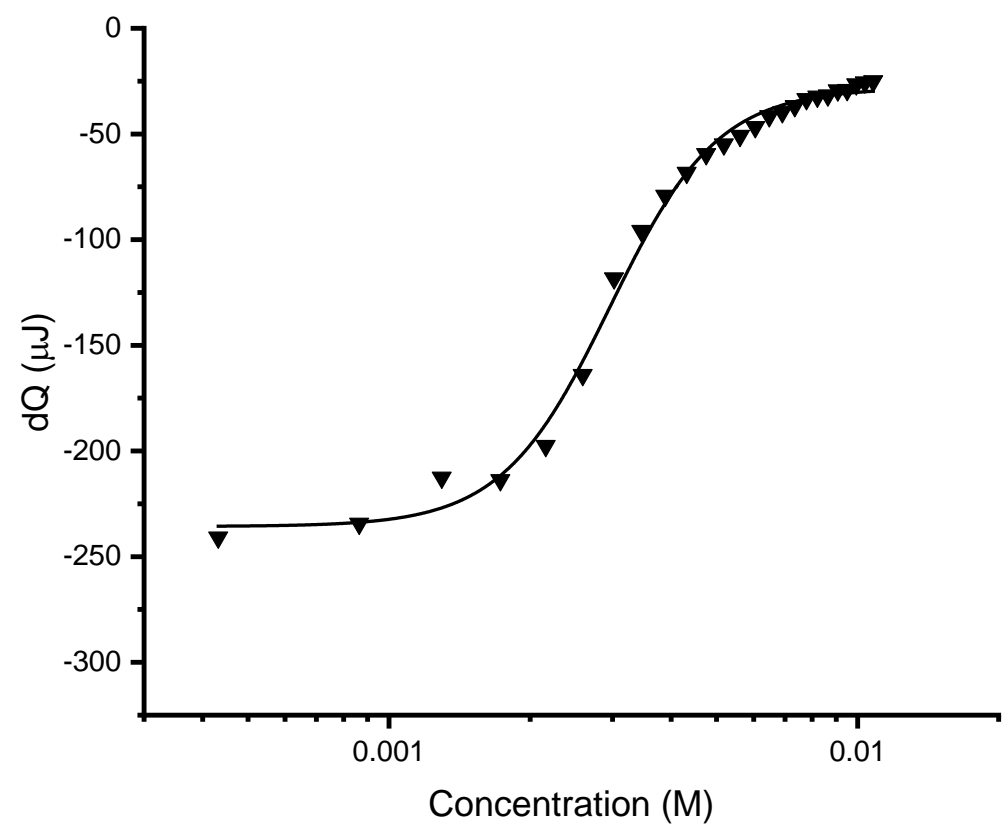

Figure S4. ITC plot used to determine the CMC of lactosyl-C-2-aminoundecane, $3 \mathbf{c}$. 
Table S1. Microbial inhibition and antibacterial activity of the C-glycoside 2-aminoundecanes. $\mathrm{OD}_{600}$ as a percentage of the untreated control.

Microorganism

P. aeruginosa

E. coli

E. amylovora

B. subtilis

P. aeruginosa

E. coli

E. amylovora

B. subtilis

P. aeruginosa

E. coli

E. amylovora

B. subtilis
Glucosyl C-2-aminoundecane, 1c

\begin{tabular}{lllllllll}
$\frac{0.17 \mathrm{mM}^{\mathrm{a}}}{2}$ & & \multicolumn{2}{c}{$0.33 \mathrm{mM}$} & \multicolumn{2}{c}{$\frac{0.67 \mathrm{mM}}{2}$} & \multicolumn{2}{c}{$1.33 \mathrm{mM}$} \\
$\mathrm{t}_{0}$ & $\mathrm{t}_{7 \mathrm{~h}}$ & $\mathrm{t}_{0}$ & $\mathrm{t}_{7 \mathrm{~h}}$ & $\mathrm{t}_{0}$ & $\mathrm{t}_{7 \mathrm{~h}}$ & $\mathrm{t}_{0}$ & $\mathrm{t}_{7 \mathrm{~h}}$ \\
33.3 & 94.3 & 0.2 & 96.5 & 0.06 & 22.5 & & \\
92 & 99 & 66.5 & 98 & 96.6 & 93.1 & 44.7 & 42.4 \\
5.3 & 95.9 & 0.4 & 8.3 & & & & \\
6.0 & 12.6 & 2.4 & 1.4 & 1.2 & 2.0 & &
\end{tabular}

Maltosyl C-2-aminoundecane, 2c

\begin{tabular}{|c|c|c|c|c|c|c|}
\hline \multicolumn{2}{|c|}{$1.4 \mathrm{mM}$} & \multicolumn{2}{|c|}{$1.8 \mathrm{mM}$} & \multicolumn{2}{|c|}{$8.9 \mathrm{mM}$} & $13.4 \mathrm{mM}$ \\
\hline & & $t_{0}$ & $\mathrm{t}_{7 \mathrm{~h}}$ & $t_{0}$ & $t_{7 h}$ & $t_{0}$ \\
\hline & & 1.6 & 3.5 & 3 & 71.4 & \\
\hline & & 2.8 & 16.6 & 3.4 & 50.4 & \\
\hline 2.3 & 39.6 & 3.4 & 3.7 & 1.8 & 3.4 & \\
\hline 1.3 & 1.6 & 1.3 & 3.4 & 2.1 & 21.7 & \\
\hline
\end{tabular}

Lactosyl C-2-aminoundecane, $3 \mathrm{C}$

\begin{tabular}{lllllllll}
$1.4 \mathrm{mM}$ & & \multicolumn{2}{l}{$1.8 \mathrm{mM}$} & \multicolumn{2}{c}{$\underline{8.9 \mathrm{mM}}$} & \multicolumn{2}{c}{$\underline{13.4 \mathrm{mM}}$} \\
& & $\mathrm{t}_{0}$ & $\mathrm{t}_{7 \mathrm{~h}}$ & $\mathrm{t}_{0}$ & $\mathrm{t}_{7 \mathrm{~h}}$ & $\mathrm{t}_{0}$ & $\mathrm{t}_{7 \mathrm{~h}}$ \\
& 0.3 & 11.0 & 1.9 & 10.3 & & \\
26.4 & 45.9 & 1.0 & 42.5 & 1.8 & 26.0 & 1.8 & 32.4 \\
0.9 & 2.8 & 0.6 & 0.5 & 1.6 & 2.6 & & \\
& & 0.6 & 1.2 & 1.7 & 3.1 & &
\end{tabular}

${ }^{a}$ Concentration of surfactant added at the time of inoculation $\left(t_{0}\right)$ and after $7 \mathrm{~h}$ of growth $\left(\mathrm{t}_{7 \mathrm{~h}}\right)$.

\section{References}

(1) Riemann, I.; Fessner, W.-D.; Papadopoulos, M. A.; Knorst, M. C-glycosides by aqueous condensation of dicarbonyl compounds with unprotected sugars. Aust. J. Chem. 2002, 55 (2), 147-154.

(2) Rodrigues, F.; Canac, Y.; Lubineau, A. A convenient, one-step, synthesis of $\beta$--glycosidic ketones in aqueous media. Chem. Commun. 2000, (20), 2049-2050. 